\title{
Hydrodynamic Modeling of Turbulence Modulation by Particles in Swirling Gas-Particle Two- Phase Flow
}

\author{
Yang Liu ${ }^{1 *}$,Lixiang Jiang ${ }^{2}$, Yongju Zhang ${ }^{1}$
}

1. College of Aerospace Engineering, Taizhou University, Zhejiang, China 318000

2. Beijing Institute of Spacecraft Environmental Engineering, Beijing, China 10094

\section{Corresponding Authors}

Dr. Yang Liu - College of Aerospace Engineering, Taizhou University, Zhejiang, 318000 China, Email: liuya@mail.tsinghua.edu.cn

\section{Authors}

Dr. Lixiang Jiang- Beijing Institute of Spacecraft Environmental Engineering, Beijing, China 10094

Email: yliu62@tzu.edu.cn

Dr. Yongju Zhang - College of Aerospace Engineering, Taizhou University, Zhejiang, China 318000 Email: yongjuzhang2019@gmail.com

\section{Author Contributions:}

The manuscript was written through the contributions of all authors. All authors have approved the revised version of manuscript. 


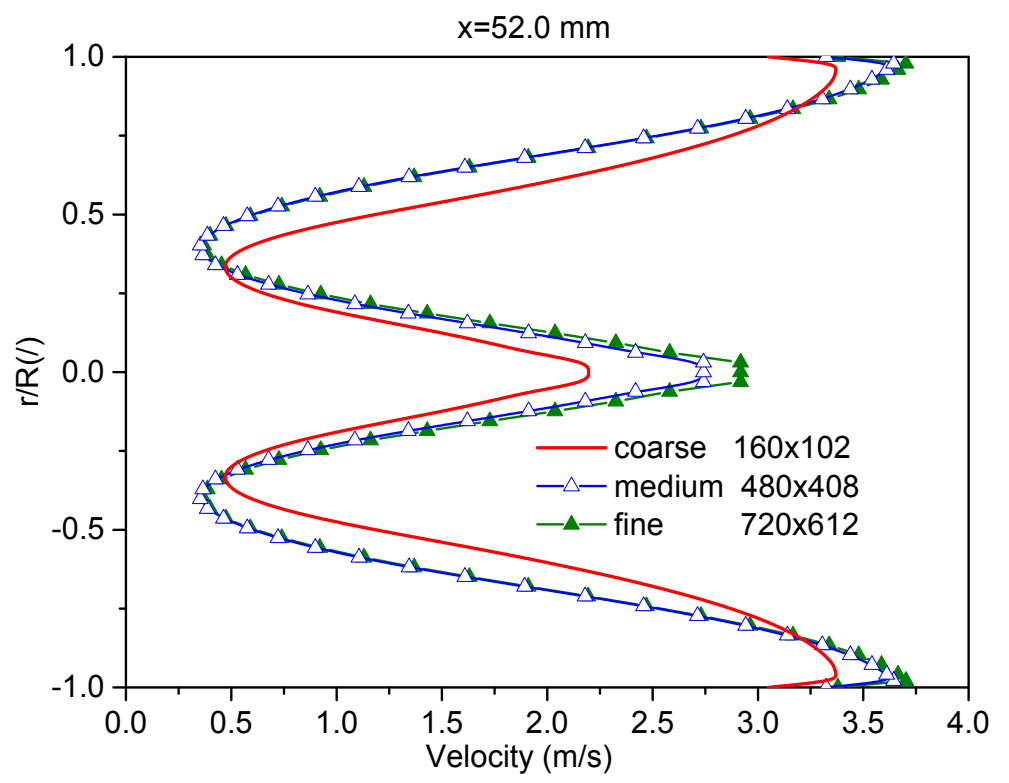

Figure S1. Grid independences of the axial particle velocity using coarse $(6.0 \mathrm{~mm} \times 1.9 \mathrm{~mm})$, medium $(2.0 \mathrm{~mm} \times$ $0.48 \mathrm{~mm})$ and fine $(1.3 \mathrm{~mm} \times 0.33 \mathrm{~mm})$ sizes 


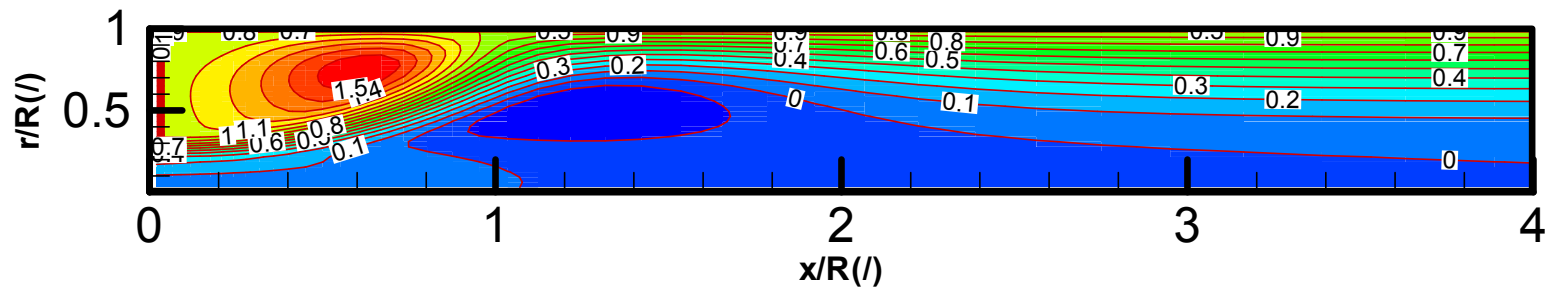

(a) gas streamline

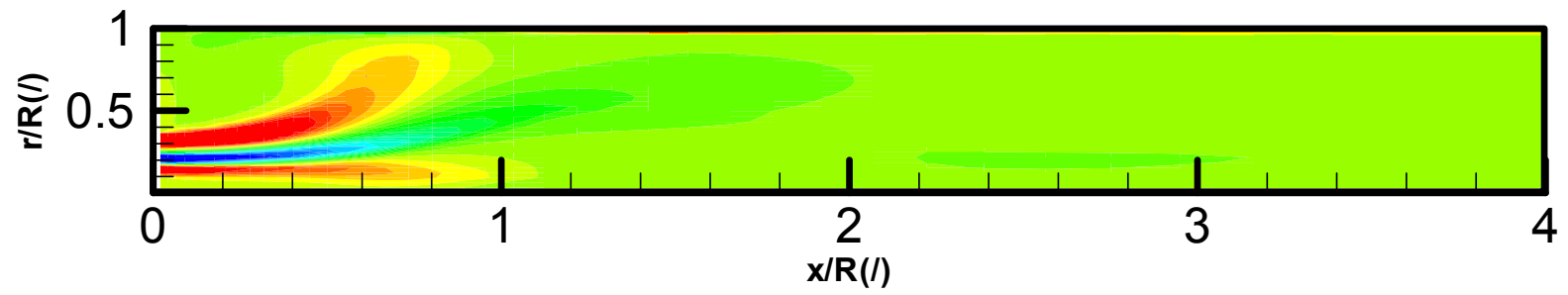

(b) gas vortices

Figure S2 Modulations of gas streamline and vortices by heavy particle, $d_{s}=160.0 \mu \mathrm{m} \mathrm{St}=1.11$ :

(a)gas streamline; and (b) gas vortices 


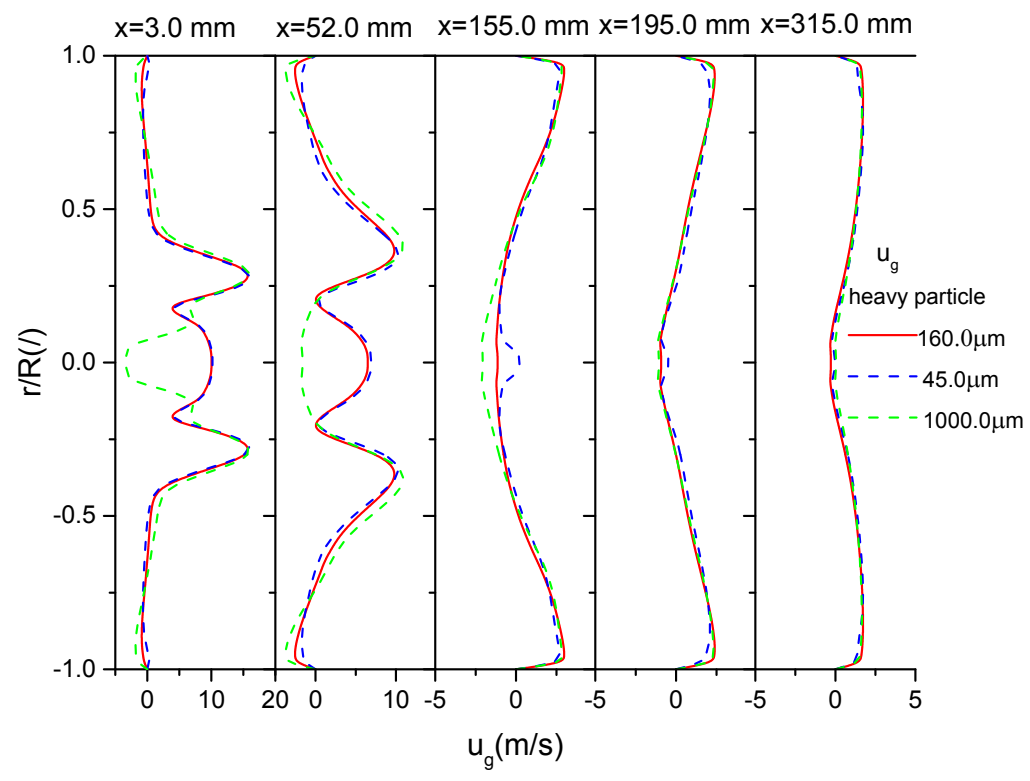

(a) axial direction

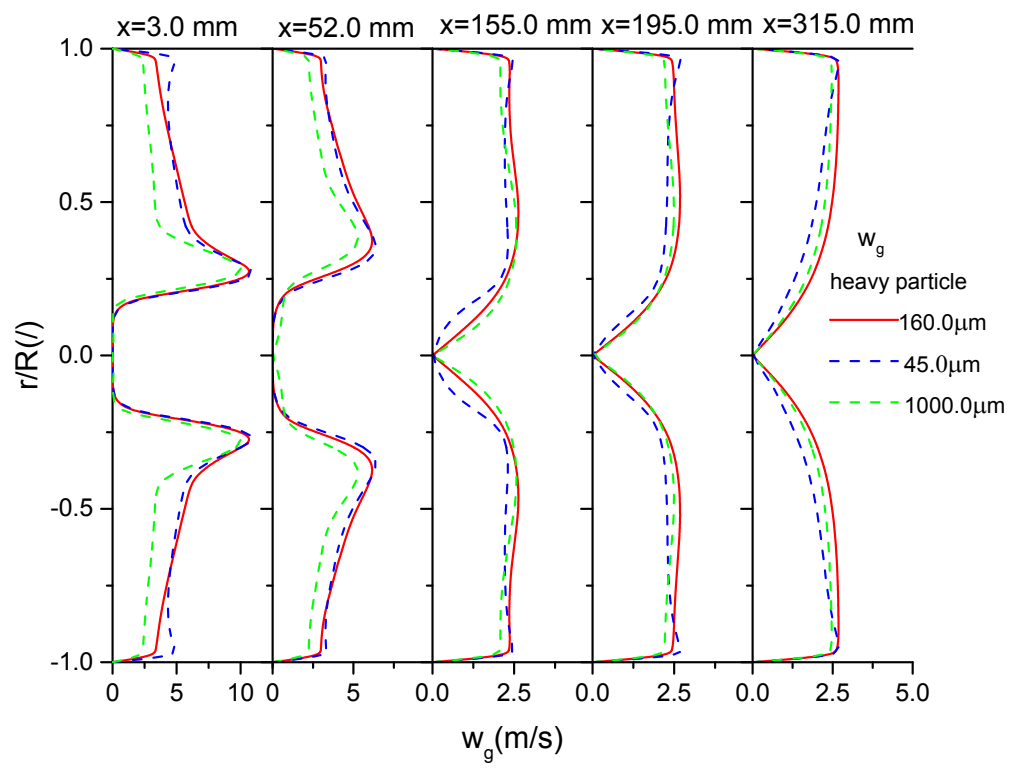

(b) tangential direction

Figure S3 Modulations of gas mean velocities by heavy particle, $\mathrm{ds}=160.0 \mu \mathrm{m}, \mathrm{St}=1.11$ :

(a) axial direction; and (b) tangential direction. 


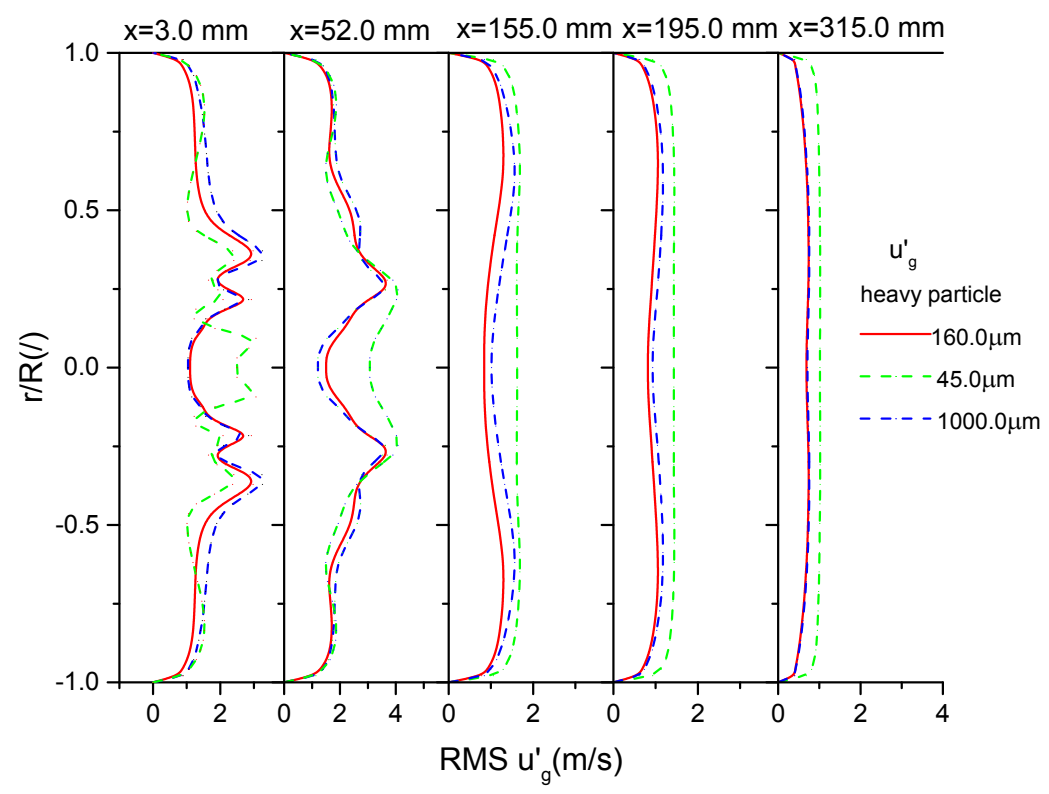

(a) axial direction

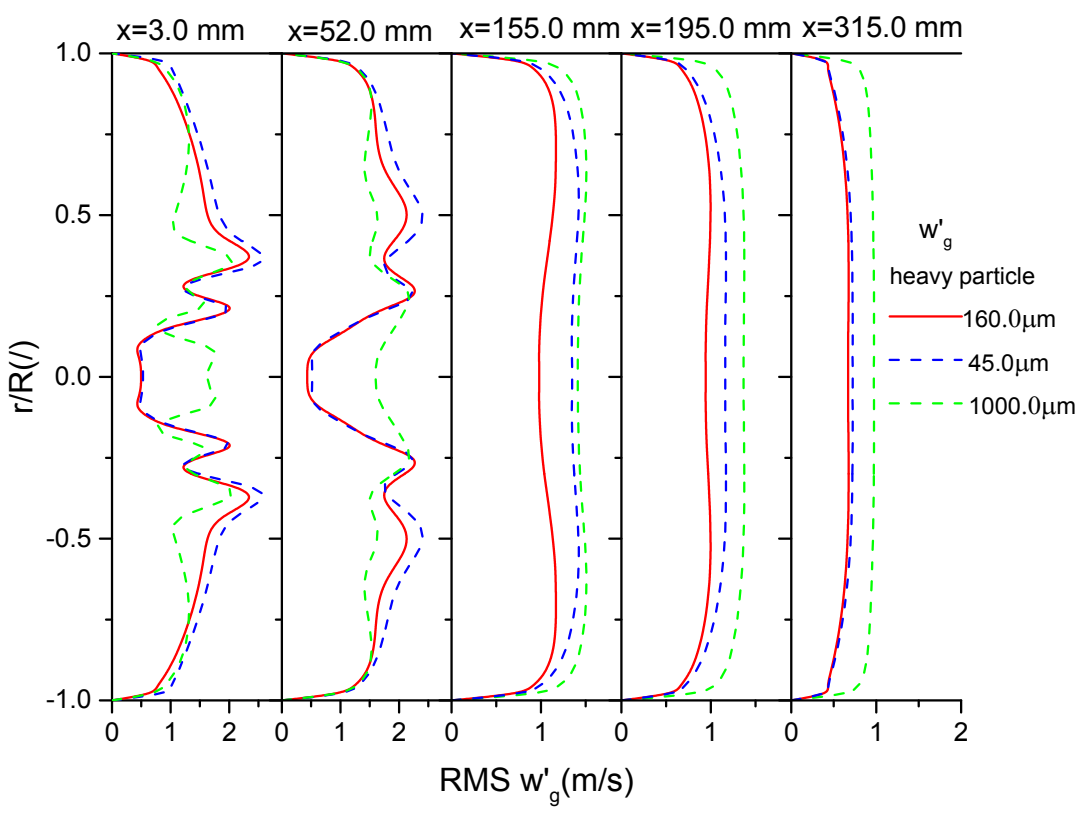

(b) tangential direction

Figure S4 Modulations of gas RMS fluctuation velocities by heavy particle, $\mathrm{ds}=160 \mu \mathrm{m} S \mathrm{St}=1.11$ : (a) axial direction; and (b) tangential direction. 


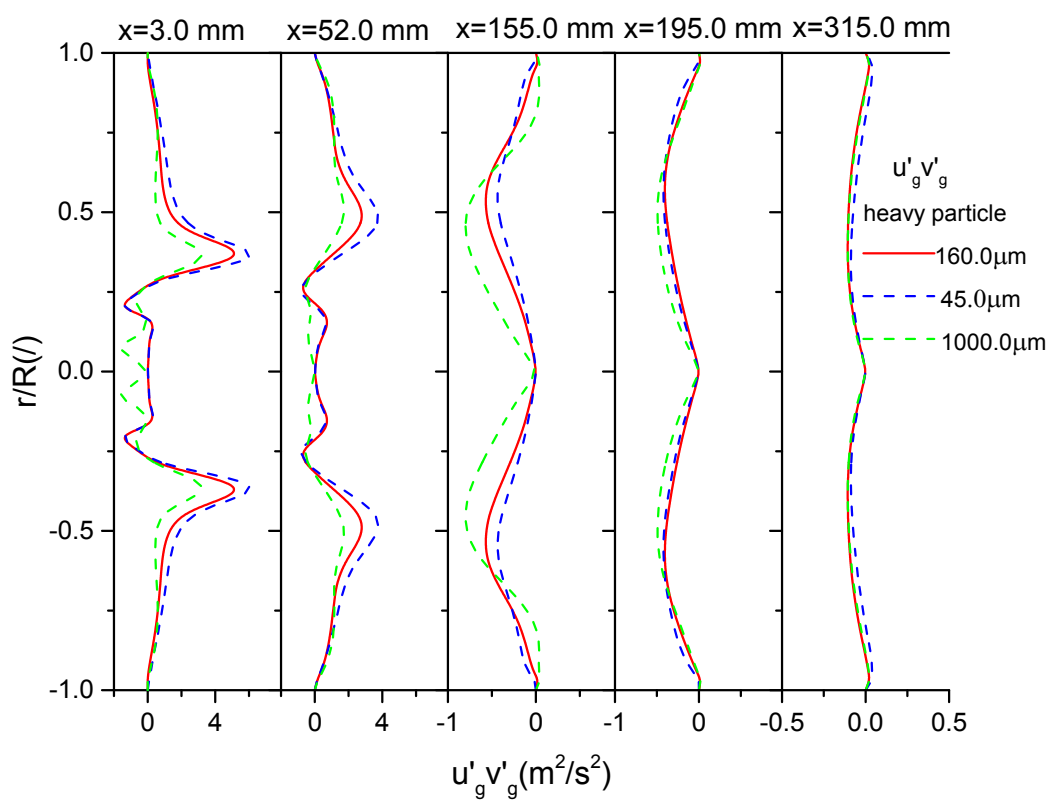

(a) axial- radial shear stress

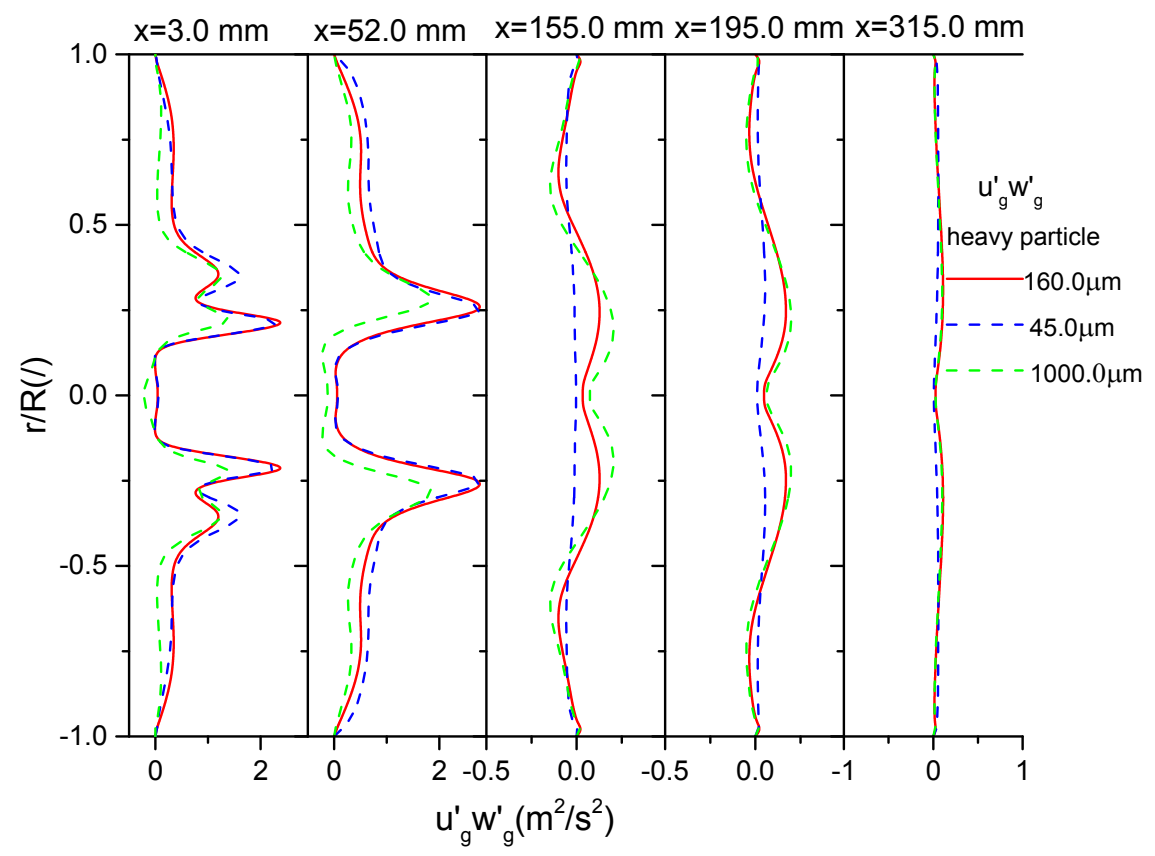

(b) axial-tangential shear stress

Figure S5 Modulations of gas shear stresses by heavy particle, $\mathrm{ds}=160.0 \mu \mathrm{m} \mathrm{St}=1.11$ : (a) axial- radial shear stress; and (b)axial-tangential shear stress. 